INPLASY

PROTOCOL

To cite: Meng et al.

Moxibustion for Nonalcoholic Fatty Liver Disease: A protocol for systematic review and meta-analysis. Inplasy protocol 2021100084. doi:

10.37766/inplasy2021.10.0084

Received: 22 October 2021

Published: 22 October 2021

Corresponding author:

Zhenzhen Meng

mzz08051005@163.com

Author Affiliation:

Guangdong Pharmaceutical

University; The First Affiliated

Hospital of Guangdong

Pharmaceutical University.

Support: None.

Review Stage at time of this submission: Preliminary searches.

Conflicts of interest:

None declared.

\section{Moxibustion for Nonalcoholic Fatty Liver Disease: A protocol for systematic review and meta-analysis}

Meng, Z1; Liu, X2; Chen, H3; Piao, S4; Guo, J5.

Review question / Objective: This review will provide a comprehensive assessment of the effectiveness and safety of moxibustion in the treatment of non-alcoholic fatty liver disease (NAFLD).

Condition being studied: NAFLD has become the most common liver disease worldwide. The overall prevalence of NAFLD worldwide continues to increase. The current treatment strategy for NAFLD is mainly aimed at lifestyle intervention and controlling the risk of metabolism and complications. Finding an effective and safe interventional therapy is urgent because there is no specific treatment method yet. Recent studies have found moxibustion also have a certain effect on the treatment of NAFLD.

INPLASY registration number: This protocol was registered with the International Platform of Registered Systematic Review and Meta-Analysis Protocols (INPLASY) on 22 October 2021 and was last updated on 22 October 2021 (registration number INPLASY2021100084).

\section{INTRODUCTION}

Review question / Objective: This review will provide a comprehensive assessment of the effectiveness and safety of moxibustion in the treatment of nonalcoholic fatty liver disease (NAFLD).
Condition being studied: NAFLD has become the most common liver disease worldwide. The overall prevalence of NAFLD worldwide continues to increase. The current treatment strategy for NAFLD is mainly aimed at lifestyle intervention and controlling the risk of metabolism and complications. Finding an effective and 
safe interventional therapy is urgent because there is no specific treatment method yet. Recent studies have found moxibustion also have a certain effect on the treatment of NAFLD.

\section{METHODS}

Participant or population: NAFLD patients diagnosed in accordance with the guidelines will be included, without limitation of age, gender and race.

Intervention: The main intervention methods include moxibustion.

Comparator: The control group included any type of control treatments except for moxibustion, such as Chinese herbal medicine, Western medicine, as well as health education, or no treatment.

Study designs to be included: Trials that compared moxibustion combined with traditional Chinese medicine as the active intervention in the treatment group conventional medicines will be included.Randomized controlled trials (RCTs) will be included.

Eligibility criteria: (1)NAFLD patients diagnosed in accordance with the guidelines will be included, without limitation of age, gender and race; (2)Interventions: The experimental group was administered moxibustion combined with or without conventional treatment. (3) Comparison: the control group was treated with health education, or no treatment or Conventional treatment.; (4)Study design: randomized controlled trials will be included; (5) Outcome indicators: total effective rate, main blood lipid indexes (TC, TG), main liver function indexes (ALT, AST).

Information sources: The two researchers independently searched and extracted pieces of literature from PUBMED, Web of Science, Cochrane Library, Embase, SinoMed, China National Knowledge Infrastructure (CNKI), China Science and Technology Journal Database, and Wanfang Database after selecting keywords and matching free words. The search period is from the database establishment to October 2021, regardless of language. The search strategy will take the form of medical subject headings (MeSH) and keywords and optimize the search strategy according to the characteristics of different databases. Keywords include: "moxibustion", "nonalcoholic fatty liver disease","non-alcoholic steatohepatitis", "randomized controlled trial", "clinical efficacy", etc.

Main outcome(s): The main outcomes will include total effective rate, main blood lipid indexes (TC, TG), main liver function indexes (ALT, AST).

Quality assessment / Risk of bias analysis: The risk of bias of the included articles is based on the RCT bias risk assessment criteria provided by the Cochrane Collaborative Group. Random sequence generation, allocation hiding, blinding of participants and personnel, blinding of outcome evaluation, incomplete and selective outcome data are made by two researcher independently Report for evaluation, discrepancies will be resolved through discussion or referral to a third examiner.

Strategy of data synthesis: The results of the included studies will be descriptively synthesized, including participant characteristics and intervention details. We will calculate the effect size by weighted average/standard average deviation (for continuous results) and $95 \% \mathrm{Cl}$ odds ratio (OR) and $95 \% \mathrm{Cl}$ (for binary results). ChiSquared test and 12 test were used to test the heterogeneity of the included literature. When $P>0.1$ and $12<50 \%$, it indicates that there is no statistical heterogeneity between the studies; conversely, when P50 $\%$, it is considered that there is statistics heterogeneity between the studies. Use RevMan5.3 provided by the Cochrane Collaboration Network for meta-analysis. If there is clinical and methodological heterogeneity, a subgroup analysis will be performed to explore the source of the heterogeneity in the study. 
Subgroup analysis: If sufficient trials are included, we will use subgroup analyses to explore potential sources of heterogeneity by age, duration of disease and interventions or other factors that need to be considered.

Sensitivity analysis: We plan to conduct sensitivity analysis by deleting individual trials one by one and repeating metaanalysis to explore the reasons for the heterogeneity between studies.

Country(ies) involved: China.

Keywords: Moxibustion; Non- Alcoholic Fatty Liver Disease; Meta-analysis; randomized controlled trials.

Contributions of each author:

Author 1 - Zhenzhen Meng.

Author 2 - Xiaomin Liu.

Author 3 - Hui Chen.

Author 4 - Shenghua Piao.

Author 5 - Jiao Guo. 